\title{
Adaptive Synergies for a Humanoid Robot Hand
}

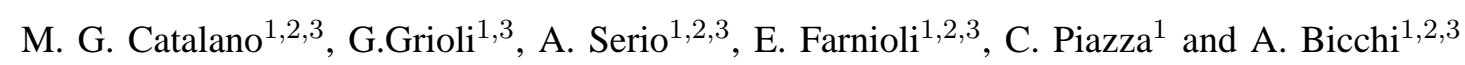

\begin{abstract}
One of the motivations behind the development of humanoid robots is the will to comply with, and fruitfully integrate in the human environment, a world forged by humans for humans, where the importance of the hand shape dominates prominently.

This paper presents the novel hand under-actuation framework which goes under the name of synergies. In particular two incarnations of this concept are considered, soft synergies and adaptive synergies. They are presented and their substantial equivalence is demonstrated.

After this, it presents the first implementation of THE UNIPI-hand, a prototype which conciliates the idea of adaptive synergies for actuation with an high degree of integration, in a humanoid shape. The hand is validated experimentally through some grasps and measurements. Results are reported also in the attached video.
\end{abstract}

\section{INTRODUCTION}

Among the many aspects which characterize the human form, distinguishing it from other animated entities, the hand stands out. Its wonderful complication gives humans a wide spectrum of possibilities, which, very roughly, can be summarized in the 4 functional groups of sensation, holding, manipulation and communication. Over the centuries of human cultural and technological evolution the importance of the hand shape asserted, as a matter of facts, by effectively shaping the word around us in a reality made of handles, knobs, buttons and keyboards, just to name some. Nowadays the development of robots in a humanoid form is also driven by the will to comply with, and fruitfully integrate in, the human environment, therefore in such hand-forged world.

Even a humanoid robot can be designed to accomplish just a small subset of all the actions usually executed by a human, for this it can resort to a simplified limb as long as it is able to perform a minimum subset of the typical hand functions. A hand designed with the only purpose of holding, for example, can still be fruitfully employed to perform a large number of everyday actions. In [1] two job activities are considered in detail as case-study (professional house-holding and machine operation). Highlithing that more than $50 \%$ of time the hand is used for grasping $(63 \%$ for the house holder and $56 \%$ for the machinist).

Focusing on grasping, [2] shows that most of the grasping hand movement can be explained, in terms of statistical variance, just by the first two or three principal components or synergies. A direct consequence of [2], was a new boost to the development of under-actuated and simplified hands,

\footnotetext{
${ }^{1}$ Centro Interdipartimentale di Ricerca "E. Piaggio", Università di Pisa, 1 Largo L. Lazzarino, 56100 Pisa, Italy.

${ }^{2}$ Advanced Robotics Department, Italian Institute of Technology, $30 \mathrm{Via}$ Morego, 16163 Genova, Italy.

3 \{m.catalano, g.grioli, a.serio, e.farnioli,

bicchi\}@centropiaggio.unipi.it
}

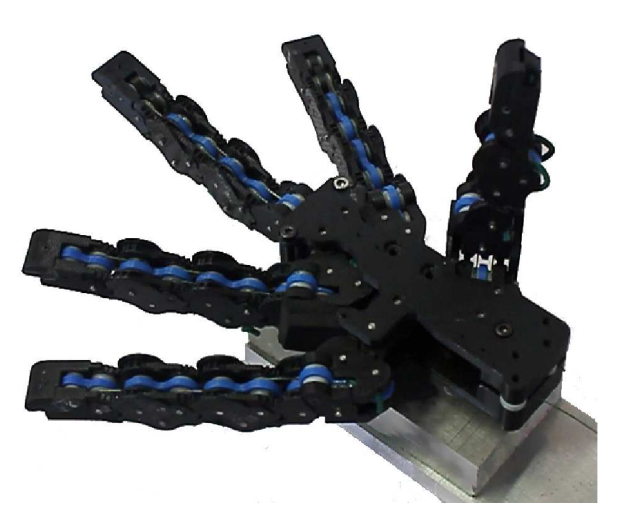

Fig. 1. THE UNIPI hand, a self-contained humanoid robotic hand powered by adaptive synergies.

as that proposed in [3], which first exploited the idea of synergies in a mechanical way.

The synergy framework finds application in hand control, both for posture definition [4] and force/impedance management [5] and has, henceforth, developed theoretically with the introduction of soft synergies first, [6], and more recently, with adaptive synergies [7], where a preliminary mechanical implementation was proposed. Nevertheless, to easily comply and fruitfully integrate in a world developed after and around human hands, non-human shaped grippers, as that proposed in [7], still need to overcome possible geometric mismatches in order to achieve performance comparable to that of humanoid hands.

This paper, retraces the concepts of soft and adaptive synergies within the rigorous twist notation framework, [8]. This allows to demonstrate their substantial equivalence, clearly stating the conditions in which a soft-synergy hand behaves like an adaptive-synergy one and vice-versa.

Later on, we present the first implementation of THE UNIPI-hand, a prototype which conciliates the idea of adaptive synergies for actuation with an high degree of integration, thus yielding a human-shaped robotic hand with adaptive synergies, whose grasping performance are shown in some experiments.

Section II of the paper retraces the evolution of adaptive synergies highlighting the differences with the different actuation approaches conceived for robot hands. Section III presents the evolution of the design of hand prototypes implementing adaptive synergies, from the preliminary proofof-concept of [7] to THE integrated human-shaped UNIPIhand, giving some details about the hand kinematics and mechanics. In section IV the grasping capabilities of the proposed hand are demonstrated by reporting some experimental 
results, which are also presented in the accompanying video. Finally conclusions are drawn in section V.

\section{Hand Actuation, AdAptation And Synergies}

\section{A. Fully Actuated Hands}

Before introducing under-actuation, we briefly present the equations describing a grasp made by a completely actuated robotic hand. This section shows how the movement of the whole system can be described knowing the joint displacement.

The notation adopted in this section is explained in Table I. More details about the analytical description of the grasp can be found in [9].

Let us consider the equilibrium equation for the grasped object. Let $w_{e}^{o} \in \mathbb{R}^{6}$ be the external wrench acting on the object and $f_{h}^{o} \in \mathbb{R}^{c}$ be the forces that the hand exerts on the object, where the dimension $c$ depends on the number and type of contact points. Introducing the grasp matrix ${ }^{\circ} G \in$ $\mathbb{R}^{6 \times c}$ referred to reference frame attached to the object, the equilibrium condition is verified when

$$
w_{e}^{o}+{ }^{o} G f_{h}^{o}=0 .
$$

Since the grasp matrix is constant, by differentiation of (1) it follows that, for the first order variation of the equilibrium configuration, it holds

$$
\delta w_{e}^{o}+{ }^{o} G \delta f_{h}^{o}=0 .
$$

Similarly, the equilibrium equation for the hand relates the contact forces with the joint torques $\left(\tau \in \mathbb{R}^{\sharp q}\right)$ by the equation

$$
\tau={ }^{o} J^{T} f_{h}^{o},
$$

where ${ }^{o T} \in \mathbb{R}^{\sharp q \times c}$ is the transpose of the hand Jacobian matrix. It is worth observing that, since the contact forces are expressed in a frame attached to the object, the Jacobian matrix depends both on the hand configuration $q \in \mathbb{R}^{\sharp q}$ and the object configuration $u \in \mathbb{R}^{6}$. The general relationship describing a perturbation of the system can be found differentiating the equation (3), obtaining

$$
\delta \tau=\bar{\Omega} \delta q+\bar{U} \delta u+\bar{J}^{T} \delta f_{h}^{o},
$$

where $\Omega=\frac{\partial^{o} J^{T} f_{h}^{o}}{\partial q} \in \mathbb{R}^{\sharp q \times \sharp q}$ and $U=\frac{\partial^{o} J^{T} f_{h}^{o}}{\partial u} \in \mathbb{R}^{\sharp q \times 6}$.

The hand/object interaction can be described introducing a virtual spring at the contact points. A force variation occurs in presence of a hand or object contact point displacements, that is

$$
\delta f_{h}^{o}=K_{c}\left({ }^{o} \bar{J} \delta q-{ }^{o} G^{T} \delta u\right),
$$

where $K_{c} \in \mathbb{R}^{c \times c}$ is the contact stiffness matrix.

Equations (2), (4) and (5) can be grouped the system

$$
\left[\begin{array}{ccccc}
I_{\sharp w} & 0 & { }^{o} G & 0 & 0 \\
0 & I_{\sharp \tau} & -\bar{J}^{T} & -\bar{\Omega} & -\bar{U} \\
0 & 0 & I_{\sharp f} & -K_{c}{ }^{o} \bar{J} & K_{c}{ }^{o} G^{T}
\end{array}\right]\left[\begin{array}{c}
\delta w_{e}^{o} \\
\delta \tau \\
\delta f_{h}^{o} \\
\delta q \\
\delta u
\end{array}\right]=0,
$$

\begin{tabular}{r|l}
\hline Notation & Definition \\
$\bar{x}$ & variation of variable $x$ \\
$\sharp x$ & value of $x$ in the reference configuration \\
\hline$c$ & number of contact constraints \\
$q \in \mathbb{R}^{\sharp q}$ & joint configuration \\
$\tau \in \mathbb{R}^{\sharp q}$ & joint torque \\
$f_{h}^{o} \in \mathbb{R}^{c}$ & contact forces exerted by the hand on the object \\
$u \in \mathbb{R}^{6}$ & pose of the object frame \\
\hline$q_{r} \in \mathbb{R}^{\sharp q}$ & reference joint configuration \\
$\sigma \in \mathbb{R}^{\sharp \sigma}$ & soft synergy configuration \\
$\varepsilon \in \mathbb{R}^{\sharp \sigma}$ & soft synergy forces \\
\hline$z \in \mathbb{R}^{\sharp z}$ & adaptive synergy displacements \\
$\eta \in \mathbb{R}^{\sharp z}$ & adaptive synergy forces \\
\hline${ }^{o} G \in \mathbb{R}^{6 \times c}$ & grasp matrix in object frame \\
${ }^{o} \in \mathbb{R}^{c \times \sharp q}$ & hand Jacobian matrix in object frame \\
$S \in \mathbb{R}^{\sharp q \times s}$ & soft synergy matrix \\
$R \in \mathbb{R}^{\sharp z \times \sharp q}$ & adaptive synergy matrix \\
& NoTATION FOR GRASP ANALYSIS.
\end{tabular}

that appears as a linear and homogeneous system of equations in the form $A \delta y=0$, where $A \in \mathbb{R}^{r_{a} \times c_{a}}$ and $\delta y \in \mathbb{R}^{c_{a}}$ is the vector containing all the system variables. It is easy to find that the nullspace basis of matrix $A$ has a number of columns equal to $c_{a}-r_{a}=\sharp w-\sharp q$. Since that, we can describe the perturbed configuration of the system knowing the external wrench variation and the displacements of the joint configuration. A formal method to obtain these relationships consists in acting on the coefficient matrix by the elementary Gauss operation for a block partitioned matrix. The final result of the procedure is a set of equations of the type

$$
\delta y_{i}=W_{i} \delta w_{e}^{o}+Q_{i} \delta q,
$$

where $\delta y_{i}{ }^{1}$ is one of the variables in $\delta y$. The matrices $W_{i}$ and $Q_{i}$ are functions of the elements of the matrix $A$, their explicit form is here omitted for brevity. From (7) it trivially follows that, without an external wrench variation ${ }^{2}$, all the variables can be found as a function of the joint displacements as

$$
\delta y_{i}=Q_{i} \delta q
$$

\section{B. Simplicity in Control}

As hinted by neuroscience works, as [2], the brain controls the human hand as a whole. Particular patterns of muscular activation give rise to organized movements, which form a base set resembling the concept of basis of a vector spac [10], that is a minimal number of linearly independent elements that, under specific operations, generate all members of the given set. Such basis is referred to as the space of postural synergies, or eigengrasp space [4], [11]. What brings out the bio-aware synergy space from other possible choices for the base to describe the hand configuration is the astounding

\footnotetext{
${ }^{1}$ A general algorithm to obtain the equations in (7) starting from (6), called GEROME-B, was presented in [9].

${ }^{2}$ The absence of the external wrench is not a general assumption. However its contribution is negligible in order to study the controllability of the
} system by the hand with or without underactuation. 
result that most of the hand grasp posture, actually $80 \%$, is statistically explained by the first two synergies alone (and $87 \%$ by the first three). Similar results can be found regarding grasping forces, as shown in [11]. This renders the synergy space a preferable base for simplification. There already exists some robotics application in robotics which take advantage of the idea of synergies. To simplify control, software synergies can be simulate on a fully actuated robotic hands (as that of Fig. 1(a)), as suggested by [4]. This can highly simplify the design phase of a grasp, by reducing the number of control variables.

The basic idea behind the use of synergies in robotics consists in specifying a suitable base for the joint space movements, called synergy matrix, $S \in \mathbb{R}^{\sharp q \times \sharp \sigma}$, where $\sharp \sigma$ is the number of synergies used. In this scenario, a hand configuration can be described in the synergy space by the vector $\sigma \in \mathbb{R}^{\sharp \sigma}$, with $\sharp \sigma \leq \sharp q$, as

$$
q=S \sigma \text {. }
$$

The possible applications of the synergy concept are not limited to software. Simplified robotic hands can be built, which embed hardware synergies in their mechanics to reduce the number of motors used to achieve most grasping tasks. The hand design by Asada [3], adopts two interchangeable set of pulleys to move the hand along two synergies, as in the simplified scheme of Fig. 1(d).

In reducing the number of control variables/motors, both approaches [4] and [3] need to confront with the gap between the number of hands DoFs (Degrees of Freedom) and the number of actuated synergies. Projecting a generic grasp configuration on the lower-dimensional sub-space spanned by $S$ implies some error in achieving the desired pose. The software synergy approach of [4] faces this by stopping the motion of each finger when it comes in contact with the grasped object, while a complementary actuation system, realized with memory-shape alloys, is proposed in [12] by the same authors of [3] to compensate for lacking DoAs (Degrees of Actuation).

\section{Soft Synergies}

An alternative to the former solutions is proposed by the introduction of soft synergies in [6]. Here synergies define the reference configuration of the hand (called virtual hand), toward which the real hand is attracted by a stiffness model. To describe this situation, for each joint we introduce a reference variable $q_{r} \in \mathbb{R}^{\sharp q}$, such that its displacements is given by

$$
\delta q_{r}=S \delta \sigma
$$

The difference between the reference value and the real position of the hand generates the joint torque necessary to balance the repulsive forces given by contact with the grasped object. In other words, defining the matrix $K_{q}^{s} \in \mathbb{R}^{\sharp q \times \sharp q}$ as the joint stiffness matrix for the soft synergy case, for the joint torques $\tau \in \mathbb{R}^{\sharp q}$, we can write that

$$
\delta \tau=K_{q}^{s}\left(\delta q_{r}-\delta q\right)
$$

Furthermore, by kineto-static duality, letting the vector $\delta \varepsilon \in$ $\mathbb{R}^{\sharp \sigma}$ be the force variation at the synergies, it is possible to prove that

$$
\delta \varepsilon=S^{T} \delta \tau
$$

The differences between (9) and (10) highlight that a soft synergy hand retains all its kinematic DOFs, but is still able to simplify the grasp mechanics, leaving the burden of finely adjusting the $\sharp q-\sharp \sigma$ less important movements to the compliance model.

A concept scheme for the hardware implementation of this approach is shown in Fig. 2(e), where springs are used in series with a mechanism similar to that of Fig. 2(d). A software implementation of a similar approach is that implemented in [5] on the DLR HAND II, through the means of a suitable impedance controller. The soft synergylike solution of [5] still requires full hand actuation and a sophisticate impedance controller on the hand. An hardware implementation could be realized on a hyper-realistic device, as that presented in [13], but such hardware, in principle similar to the scheme of Fig. 2(b), is actually twice as complex as that of a fully actuated hand.

Soft Synergy Hand Control: the effect of the synergistic under-actuation, described by (10), (11) and (12), can be taken into account in the equations (8). In particular for $i=$ 2 , thus for variable $\tau$, we obtain simply that

$$
\delta \tau=Q_{\tau} \delta q
$$

Substituting (13) in (11) and taking into account (10), it is easy to obtain

$$
\delta q=\left(K_{q}^{s}+Q_{\tau}\right)^{-1} K_{q}^{s} S \delta \sigma
$$

Thus, from (8), it holds that

$$
\delta y_{i}=Q_{i}\left(K_{q}^{s}+Q_{\tau}\right)^{-1} K_{q}^{s} S \delta \sigma .
$$

The equation (14) and the system described by the (15) are able to give us a complete description of the variation of the hand/object configuration given the position change at the synergy level.

To describe the situation where the soft synergies are controlled by forces, assessing (15) for the variable $\tau$, placing it into (12), we can calculate the necessary force at the synergy level as

$$
\delta \varepsilon=S^{T} Q_{\tau}\left(K_{q}^{s}+Q_{\tau}\right)^{-1} K_{q}^{s} S \delta \sigma .
$$

Inverting the result we arrive to

$$
\delta \sigma=\left(S^{T} Q_{\tau}\left(K_{q}^{s}+Q_{\tau}\right)^{-1} K_{q}^{s} S\right)^{-1} \delta \varepsilon .
$$

Substituting (17) in (14), the hand joint displacement becomes

$$
\delta q=\left(K_{q}^{s}+Q_{\tau}\right)^{-1} K_{q}^{s} S\left(S^{T} Q_{\tau}\left(K_{q}^{s}+Q_{\tau}\right)^{-1} K_{q}^{s} S\right)^{-1} \delta \varepsilon .
$$

Therefore, substituting (18) in (8), the complete system variation depending on the soft synergy forces is also obtained. 

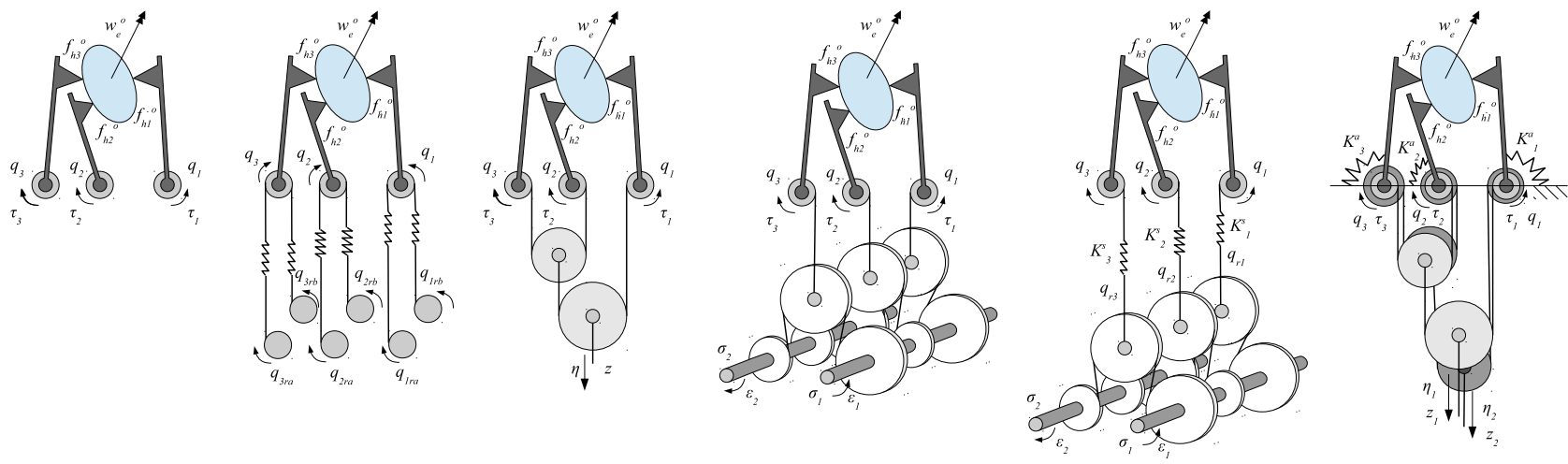

Fig. 2. A simplified three-finger hand grasping an object, with concept implementation of different actuation paradigms.

\section{Simplicity in Design}

From early approaches, as [14], adaptive under-actuated hands evolved toward simplicity in design, such as those proposed in [15], [16] and [17]. To pursue this goal adaptive under-actuated hands make use of an approach based on differential transmissions, which distribute the displacements of a very small number of motors $z \in \mathbb{R}^{\sharp z}$, with $\sharp z \leq \sharp q$, to all the fingers actuating a linear combination of $q$, as in

$$
R \delta q=\delta z .
$$

as introduced in [7], $R \in \mathbb{R}^{\sharp z \times \sharp q}$ can be designed to be an adaptive synergy matrix, composed by the transmission ratios from the actuator to each joint. The scheme of Fig.1(c) shows a concept of this pattern of actuation. Adaptive synergies go a step past soft synergies by enabling a method to effectively exploit synergies for the design of under-actuated hands, compensating for the adoption of a reduced number of synergies with the possibility to adapt to the shape of the objects to be grasped. On the other hand, we go beyond traditional adaptive hands, by proposing a technique to combine multiple DOAs on the same under-actuated hand, in a way that each DOA globally actuates the whole hand and DOAs are hierarchically ordinated by a functional bioinspired relationship.

Adaptive Synergy Hand Control: in order to find the hand/object displacements imposed by given adaptive synergies, we first look for the transmittable joint torques. Taking into account (19), from the kineto-static duality, $R$ relates the force $\eta \in \mathbb{R}^{\sharp z}$ applied by the actuators to the torque $\tau$ on the joints by

$$
\delta \tau=R^{T} \delta \eta
$$

The uniqueness of the free movement of the hand is assured by the introduction of elastic actions in parallel with the mechanical actuation system, as in Fig.1(f). Thus, defining the joint stiffness matrix for the adaptive synergy case as $K_{q}^{a} \in \mathbb{R}^{\sharp q \times \sharp q}$, we can modify (20) in

$$
\delta \tau=R^{T} \delta \eta-K_{q}^{a} \delta q
$$

Considering (21) and (13), it immediately follows that

$$
\delta q=\left(K_{q}^{a}+Q_{\tau}\right)^{-1} R^{T} \delta \eta,
$$

thus, substituting in (8), we can obtain the complete description of the hand/object displacements resulting from the application of forces at the synergy level.

To find a relationship describing the case of adaptive synergies controlled by the position, we start substituting the equation (19) in (22). Inversion allows us, to find that

$$
\delta \eta=\left(R\left(K_{q}^{a}+Q_{\tau}\right)^{-1} R^{T}\right)^{-1} \delta z
$$

which is the expression of the synergy forces as a function of the synergy displacements. This result can be placed in (22) obtaining

$$
\delta q=\left(K_{q}^{a}+Q_{\tau}\right)^{-1} R^{T}\left(R\left(K_{q}^{a}+Q_{\tau}\right)^{-1} R^{T}\right)^{-1} \delta z,
$$

thus, substituting in (8), also the complete system description depending on the adaptive synergy displacements.

\section{E. From Soft to Adaptive Synergies}

We now look for a way to obtain an adaptive synergy matrix $R$ and a joint stiffness matrix $K_{q}^{a}$ able to imitate the effects of a given soft synergy under-actuation. In this sense, we suppose to know the joint stiffness matrix $K_{q}^{s}$ and the soft synergy matrix $S$. Thus, from (14) and (18), we can suppose to know the term

$$
\hat{S}=\left\{\begin{array}{l}
\left(K_{q}^{s}+Q_{\tau}\right)^{-1} K_{q}^{s} S \\
\left(K_{q}^{s}+Q_{\tau}\right)^{-1} K_{q}^{s} S\left(S^{T} Q_{\tau}\left(K_{q}^{s}+Q_{\tau}\right)^{-1} K_{q}^{s} S\right)^{-1},
\end{array}\right.
$$

where the first holds if the soft synergies are position controlled, the second otherwise. Since the matrix $\hat{S} \in \mathbb{R} \sharp q \times \sharp \sigma$ is able to describe the joint displacements, and since from this depends all the hand/object motion, as by (8), we can say that to obtain the equivalent adaptive synergies means to obtain the same joint displacements. If we want to do that by a force controlled adaptive synergies we have to consider the 
equation (22), obtaining, under the hypothesis of $\sharp z=\sharp \sigma$, that

$$
\left(K_{q}^{a}+Q_{\tau}\right)^{-1} R^{T}=\alpha \hat{S},
$$

where $\alpha$ is a non-null coefficient able to accord units of measurement. From (26) it immediately follows that

$$
R^{T}=\alpha\left(K_{q}^{a}+Q_{\tau}\right) \hat{S},
$$

allowing us to find suitable matrices $R$ and $K_{q}^{a}$.

On the contrary, if we want to use a position controlled adaptive synergies, we have to consider (24), obtaining

$$
\left(K_{q}^{a}+Q_{\tau}\right)^{-1} R^{T}\left(R\left(K_{q}^{a}+Q_{\tau}\right)^{-1} R^{T}\right)^{-1}=\alpha \hat{S},
$$

or equivalently

$$
R^{T}=\alpha\left(K_{q}^{a}+Q_{\tau}\right) \hat{S} R\left(K_{q}^{a}+Q_{\tau}\right)^{-1} R^{T} .
$$

It is easy to prove that (29) is satisfied only if

$$
\hat{S} R=\frac{1}{\alpha} I,
$$

where $I \in \mathbb{R}^{\sharp \sigma \times \sharp \sigma}$ is an identity matrix. Unfortunately, since in general $\hat{S}$ is a tall matrix and $R$ is a fat matrix, the product $\hat{S} R$ can not be a full rank matrix, thus it is not possible to find a suitable position controlled adaptive synergy underactuation able to imitate the effects of the given soft.

\section{F. From Adaptive to Soft Synergies}

Similarly to what seen before, we can search how to introduce a soft synergy actuation which imitates a given adaptive one, in terms of hand/object controllable displacements. Under this hypothesis, we can assume to know the adaptive synergy matrix $R$ and the joint stiffness matrix $K_{q}^{a}$, that is the term

$$
\hat{R}^{T}=\left\{\begin{array}{l}
\left(K_{q}^{a}+Q_{\tau}\right)^{-1} R^{T} \\
\left(K_{q}^{a}+Q_{\tau}\right)^{-1} R^{T}\left(R Q_{\tau}\left(K_{q}^{a}+Q_{\tau}\right)^{-1} R^{T}\right)^{-1},
\end{array}\right.
$$

where the first holds in the force control case, the second otherwise. In order to find an equivalent position controlled soft synergy, starting from (14), it is easy to obtain that

$$
S=\beta\left(K_{q}^{s}\right)^{-1}\left(K_{q}^{s}+Q_{\tau}\right) \hat{R}^{T},
$$

where the coefficient $\beta$ has the same function of $\alpha$ in (26).

It is worth observing that, considering (25) for the position controlled soft synergy case, and (31) for the force controlled adaptive synergy case, from (26) and (32), in the particular case of $K_{q}=K_{q}^{s}=K_{q}^{a}$, the map between the two underactuation type is simply given by

$$
R^{T}=\alpha K_{q} S,
$$

in accordance with the results of [7].

Conversely, to find a suitable force controlled soft synergy, we have to consider the equation (18). Considerations similar to the previous allow us to easily obtain the condition

$$
\hat{R}^{T} S^{T}=\frac{1}{\beta} Q_{\tau}^{-1}
$$

Since the right hand term is a non-singular matrix, as for condition (30), it follows that even in this case it is not possible to solve the problem.

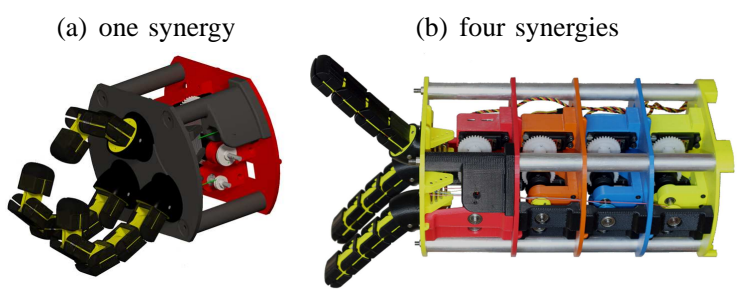

Fig. 3. Preliminary prototype of Adaptive synergy hand. The left panel shows a render of the prototype assembled with two 4-phalanxes fingers, a 2-phalanxes thumb and one adaptive synergy. The right panel shows a picture of the actual prototype, where all the fingers have three phalanxes and four adaptive synergies are implemented.

\section{A SElF-CONTAIned Humanoid Hand}

To validate the concept of adaptive synergies, a proofof-concept rapid prototype hand was designed in [7]. The prototype had three fingers and was modular with respect to the number of phalanxes in each finger and the number adaptive synergies (from one up to four). A picture of the prototype assembled with 3-phalanxes fingers and four adaptive synergies is shown in Fig. 3(a). Another possible assembly with two 4-phalanxes fingers, one 2-phalanxes thumb and one adaptive synergy is shown instead in Fig. 3(a).

In the next section we show how adaptive synergies can be successfully implemented also in the design of a humanshaped hand. Our purpose is to build a simple and robust human-shaped hand, which implements the concept of adaptive synergies, following the scheme of Fig. 2(f). The human hand has 19 DOFs, but its complexity can be approximated by a chain of 1 DOF joints (i.e. revolute joints), properly rotated to achieve flexion-extension, abduction-adduction and opposition movements. This simplified approach can be exploited to realize a hand out of only a few base modules.

The mechanical implementation of adaptive synergy dictates for the need to transfer simultaneously force and torque in a coordinated way for each DOF, implementing the transmission ratio $R$ of (19).

A revised version of Hillberry's rolling joint [18] is, in the authors view, particularly suitable to realize this type of hand.

\section{A. The Hillberry's rolling Joint.}

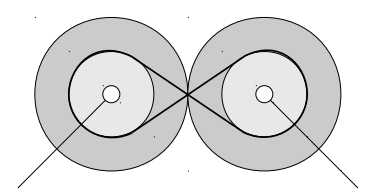

Fig. 4. Schematics of Hillberry joint. It consists of two cylinders in rolling contact on each other. Hold together by a system of bands.

A Hillberry's joint consists of two pairs of cylinders in rolling contact on each other, as schematically shown in Fig. 4. Each of these two parts can be seen as a revolute joint. The two cylinders are held together through a system 
(a) Perspective view

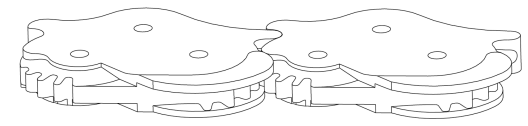

(b) Side view movement

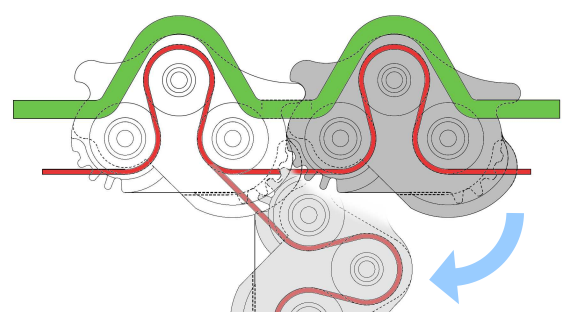

Fig. 5. Revised version of Hillberry's joint (a) and profile view from the plane orthogonal to the rolling direction (b).

of bands, which can be rigid or elastic [18]. A Hillberry's joint exhibit many advantages:

1) The particular mechanical structure allows for easy modification of its characteristic diameters, thus changing the transmission ratio of the joint and therefore the transmitted force. Zero transmitted torque can be achieved by adequate tendon routing.

2) Joint friction is low, despite the lack of bearings.

3) The rotation range is about $180^{\circ}$, that covers the needed rotation range of about $90^{\circ}$.

4) It is easily scalable.

5) Absence of mechanical connection elements, like screws and bolts, is a major simplification.

Thanks to the presence of Hillberry's joint, an actuation system relying completely on tendons was achieved. The tendon carries the actuation and ensures also mechanical locking. The intrinsic elasticity of the joint, given by the elastic bands (in green in Fig. 5), allows us to use an unidirectional actuation system. In particular, joint elasticity is determined by the bias of the elastic band from the (moving) contact point on the Hillberry's joint and can be, to some extent, adjusted by pretensioning the elastic band. The chosen implementation relies on a single cable acting on the whole hand and it gives adaptivity to the overall system, without the need for a differential gear mechanism (unlike the prototype presented [7]).

\section{B. Robustness}

In addition to the previously described features, another attribute of the joint is robustness. Indeed, in robots and humans, the hand is liable for crash, in particular during grasp and exploration movements. The designed joint is able to withstand severe disarticulations, as show in Fig. 6 , exploiting the intrinsic system elasticity. The revisited Hillberry joint shows the following features:

1) two cylindrical structures in rolling contact on each other,

2) lateral walls in each side of the joint, to ensure the following of the rolling profile, also in the case of (a) Side bend

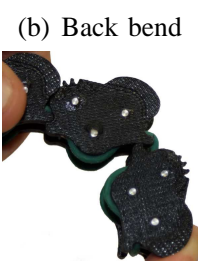

(c) Twist

(d) Skew bend
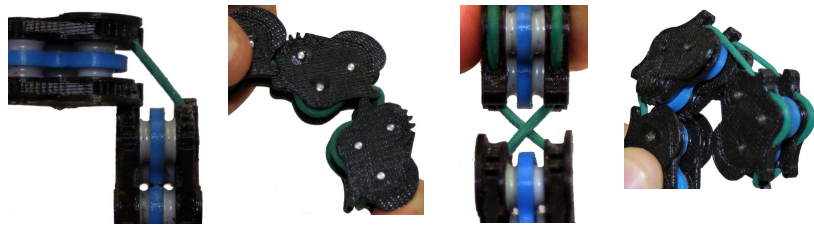

Fig. 6. Example of disarticulations the designed joint is able to withstand.

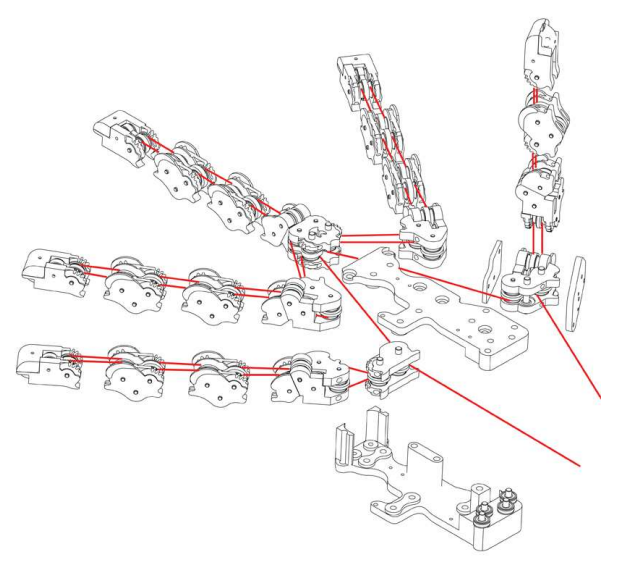

Fig. 7. Exploded view of the modules of the whole hand.

transverse external forces. The walls present a slope of about $80^{\circ}$, as shown in Fig. 5 and Fig. 6. Each lateral wall is housed in a recess of the same dimension, properly designed in the other corresponding part of the joint.

3) An elastic tendon, locked at both ends with some pretensioning, which holds together the two parts of the joint and provides elasticity $K^{a}$.

4) A matching geared coupling integrated on rolling surface to constrain rolling contact. This profile is not that of a complete gear, but it's gradual (it can be seen in Fig. 5).

5) A small profile is included in the back of the joint, to ensure correct return to the rest position in case of accidental over-opening of the joint.

6) Finally a series of ball bearings pulleys inside each joint, house the tendon actuation. The diameter of the pulleys range from $8 \mathrm{~mm}$ to $6 \mathrm{~mm}$, with a wrapping radius of about $7 \mathrm{~mm}$. A properly designed spacer, separates the pulley each from the other.

\section{Hand Description}

The whole hand is realized by the assembly of 20 modules, as showed in the exploded view of Fig. 7. In rest position, all the fingers are completely open and form an angle of about $30^{\circ}$ one with respect the other. The thumb is rotated of $90^{\circ}$ about its axis and is perpendicular to the palm, the full kinematics can be seen in Fig. 8.

Despite the integrated pulleys, friction ultimately limits, in practice, the number of joints that can be actuated by a 


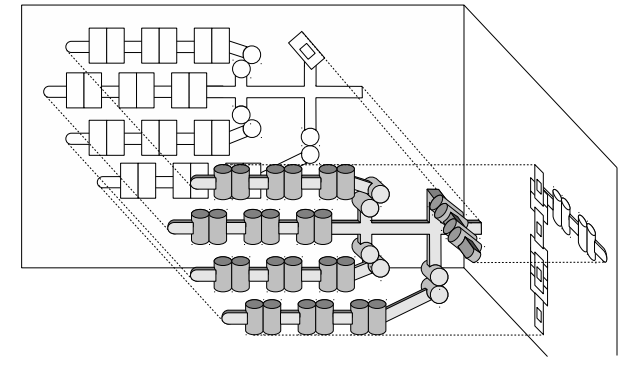

Fig. 8. Schematics of the kinematics of THE UNIPI hand. As explained in the legend, rotation joints are represented by one light gray cylinder, while rolling joints are represented by a pair of dark gray cylinders.

single motor. This phenomenon is partly contained thanks to the tendon actuation which is realized pulling the tendon from both ends, allowing the implemented hand to perform satisfactorily. Further compensation for friction comes from proper choice of pulley radii, which is designed in order to let the real hand mimicry the first human hand synergy. Design techniques for pulley radii constitute a very faceted topic which is out of the scope of this paper.

\section{EXPERIMENTAL VALIDATION}

\section{A. Experimental Setup}

The proposed has been experimentally validated by measuring the maximum grasp force and holding torque and by grasping some common objects.

All the experiments and grasps of the following paragraphs rely on a PID control implemented on the motor position. The tendon was actuated by a 516:1 $12 \mathrm{~V}$ DC gear motor, equipped with a HEDS 5540 digital encoder (1024 counts per turn) and driven by Sabertooth Syren 10 driver. Encoder signals were acquired with a PhidgetsEncoder High Speed board while driver commands were sent trough a Phidget 4-Output board. After calibration, the hand is controlled acting on the percentage of closure: from $0 \%$ (completely opened) to $100 \%$ (completely closed). In this manner it is possible to control the hand with a simple slider. For force and torque experiments we used an ATI nano $17 \mathrm{~F} / \mathrm{T}$ sensor with UDP interface to measure holding force and torque of the robotic hand. The sensor was embedded in the two test objects of Fig. 9: a split cylinder (Fig. 9(a)) to measure the grasp force and a disk (Fig. 9(b)) to measure maximum holding torque. Control and measurements were performed in MATLAB/SIMULINK. During all the experiments the hand is equipped with an off-the-shelf rubber working glove to supply good contact friction. For grasp force experiments we used a cylinder of $120 \mathrm{~mm}$ height and diameter of 45 $\mathrm{mm}$ (see also Fig. 9(a)). For holding torque experiments we used a cylinder of $20 \mathrm{~mm}$ height and diameter of $95 \mathrm{~mm}$ (see also Fig. 9(b)). In Fig. 9(a) we report forces and torque acquisitions during sensorized object grasp.

\section{B. Experimental Results}

In Fig. 10(b) we report force acquisitions during sensorized object grasp. It is possible to notice how forces (a) Grasp force test object

(b) Holding torque test object
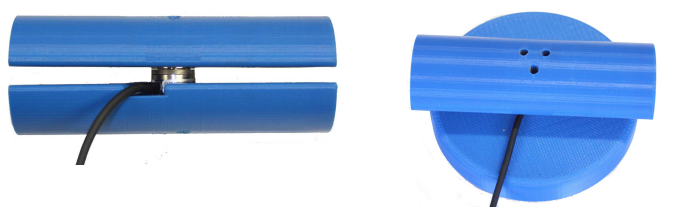

Fig. 9. Sensorized object for torque measurements (8(b)) and sensorized object for force measurements (8(a)).

(a) Torques

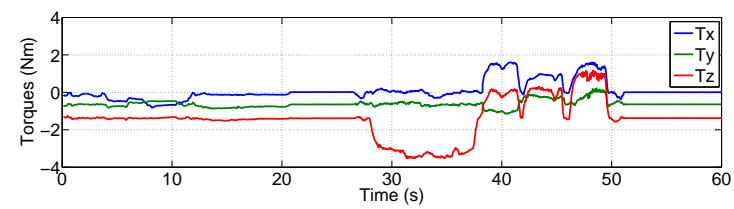

(b) Forces

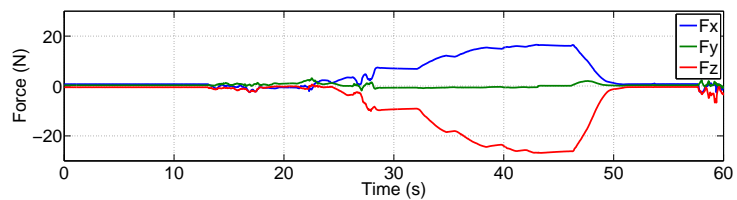

Fig. 10. Torques and forces of the robotic hand during grasp task.

increased when fingers get in contact with the sensorized cylinder (step behavior of the red and blue line in Fig. 10(b)). We achieved a maximum holding torque of $3 \div 3.5 \mathrm{Nm}$. We achieved a maximum holding force of about $25 \div 28 \mathrm{~N}$ along $z$ axis. For more details on the performed experiments, see also the attached video.

\section{Experimental Grasps}

To test the adaptiveness of the robotic hand, trial grasps of several objects with different shapes were performed. Fig. 11 shows some of this adaptive grasps. Other grasps are shown in the video footage.

\section{CONCLUSION}

This paper presented the novel hand under-actuation framework which goes under the name of synergies. After analyzing both soft and adaptive synergies within the twist notation framework and deriving mathematically the conditions which allows one to perform as the other and the conditions in which this equivalence is not possible, we present the first implementation of the UNIPI-hand, a highly integrated prototype of human hand which conciliates the idea of adaptive synergies with a human form factor. The hand is validated experimentally through some grasps and measurements, as also reported by the attached video.

\section{ACKNOWLEDGMENT}

The authors would like to thank Andrea Di Basco, Fabrizio Vivaldi, Simone Tono and Emanuele Silvestro for their valuable help in the realization of the prototypes. 
(a) Cube Grasp

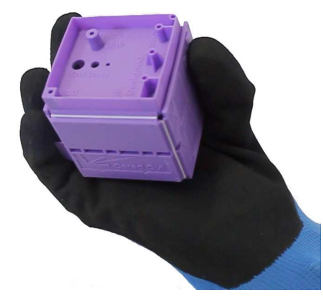

(f) Cube Dimensions

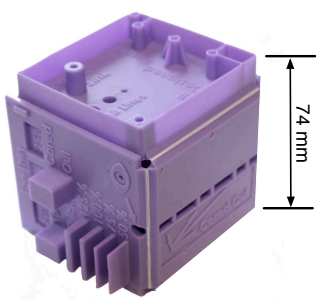

(b) Bottle Grasp

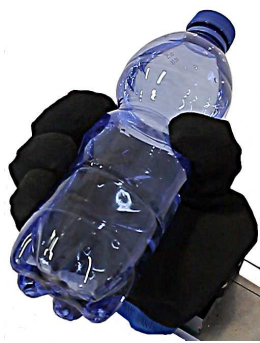

(g) Bottle Dimensions

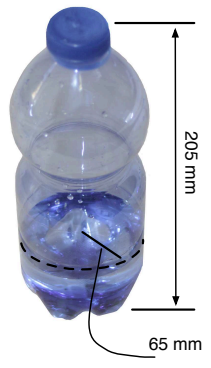

(c) Reel Grasp

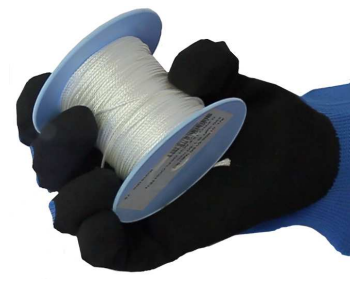

(h) Reel Dimensions

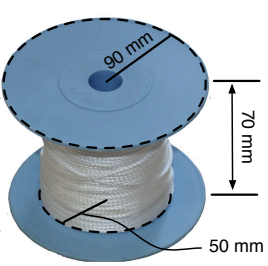

(d) Pincer Grasp

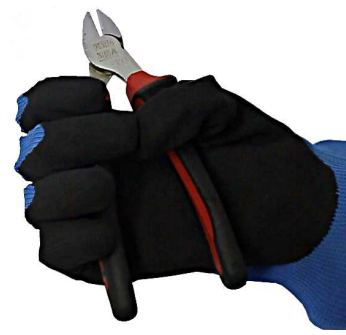

(i) Pincer Dimensions

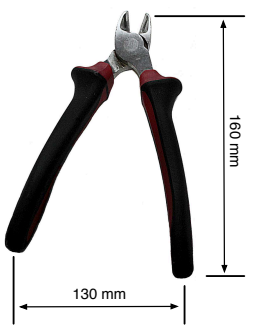

(e) Stapler Grasp

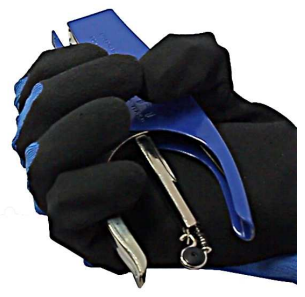

(j) Stapler Dimensions

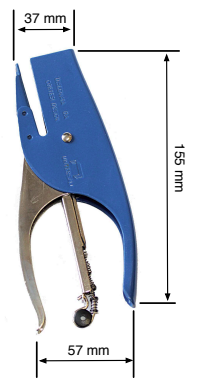

Fig. 11. Some experimental grasps performed with the proposed hand. Grasps are performed both on simple geometrical shapes (panels (a) - (b)) and on more complex shapes (panels (c) - (e)). Adaptability of the hand w.r.t the object shape is highlighted. Bottom row (panels (f) - (j)) show main object dimensions.

This work is supported by the European Commission under the CP-IP grant no. 248587 "THE Hand Embodied", within the FP7-2007-2013 program "Cognitive Systems and Robotics", ERC Advanced Grant no. 291166 "SoftHands" A Theory of Soft Synergies for a New Generation of Artificial Hands- and FP7/2007-2013 grant agreement n257462 "HYCON2 Network of excellence".

\section{REFERENCES}

[1] J. Zheng, S. De La Rosa, and A. Dollar, "An investigation of grasp type and frequency in daily household and machine shop tasks," in Robotics and Automation (ICRA), 2011 IEEE International Conference on. IEEE, 2011, pp. 4169-4175.

[2] M. Santello, M. Flanders, and J. Soechting, "Postural hand synergies for tool use," The Journal of Neuroscience, vol. 18, no. 23, pp. $10105-$ $10115,1998$.

[3] C. Brown and H. Asada, "Inter-finger coordination and postural synergies in robot hands via mechanical implementation of principal components analysis," in Intelligent Robots and Systems, 2007. IROS 2007. IEEE/RSJ International Conference on. IEEE, 2007, pp. 28772882.

[4] M. Ciocarlie, C. Goldfeder, and P. Allen, "Dexterous grasping via eigengrasps: A low-dimensional approach to a high-complexity problem," in Proceedings of the Robotics: Science \& Systems 2007 Workshop-Sensing and Adapting to the Real World, Electronically published. Citeseer, 2007.

[5] T. Wimboeck, C. Ott, and G. Hirzinger, "Passivity-based object-level impedance control for a multifingered hand," in Intelligent Robots and Systems, 2006 IEEE/RSJ International Conference on. Ieee, 2006, pp. 4621-4627.

[6] A. Bicchi, M. Gabiccini, and M. Santello, "Modelling natural and artificial hands with synergies," Philosophical Transactions of the Royal Society B: Biological Sciences, vol. 366, no. 1581, pp. 31533161, 2011.

[7] G. Grioli, M. Catalano, E. Silvestro, S. Tono, and A. Bicchi, "Adaptive synergies: an approach to the design of under-actuated robotic hands." in Intelligent Robots and Systems, 2012. IROS 2012. IEEE/RSJ
International Conference on. IEEE, 2012, p. submitted. [Online]. Available: https://dl.dropbox.com/u/5583591/SecondHand.pdf

[8] R. Murray, Z. Li, and S. Sastry, A mathematical introduction to robotic manipulation. CRC, 1994.

[9] A. B. M. Gabiccini, E. Farnioli, "Grasp and manipulation analysis for synergistic underactuated hands under general loading conditions," in Robotics and Automation (ICRA), 2012 IEEE International Conference on.

[10] T. Easton, "On the normal use of reflexes: The hypothesis that reflexes form the basic language of the motor program permits simple, flexible specifications of voluntary movements and allows fruitful speculation," American Scientist, vol. 60, no. 5, pp. 591-599, 1972.

[11] D. Prattichizzo, M. Malvezzi, and A. Bicchi, "On motion and force controllability of grasping hands with postural synergies," Proceedings of Robotics: Science and Systems, Zaragoza, Spain, 2010.

[12] J. Rosmarin and H. Asada, "Synergistic design of a humanoid hand with hybrid dc motor-sma array actuators embedded in the palm," in Robotics and Automation, 2008. ICRA 2008. IEEE International Conference on. IEEE, 2008, pp. 773-778.

[13] M. Grebenstein, A. Albu-Schaffer, T. Bahls, M. Chalon, O. Eiberger, W. Friedl, R. Gruber, S. Haddadin, U. Hagn, R. Haslinger et al., "The dlr hand arm system," in Robotics and Automation (ICRA), 2011 IEEE International Conference on. IEEE, 2011, pp. 3175-3182.

[14] R. Tomovic and G. Boni, "An adaptive artificial hand," Automatic Control, IRE Transactions on, vol. 7, no. 3, pp. 3-10, 1962.

[15] C. Gosselin, F. Pelletier, and T. Laliberte, "An anthropomorphic underactuated robotic hand with 15 dofs and a single actuator," in Robotics and Automation, 2008. ICRA 2008. IEEE International Conference on. IEEE, 2008, pp. 749-754

[16] A. Dollar and R. Howe, "The highly adaptive sdm hand: Design and performance evaluation," The International Journal of Robotics Research, vol. 29, no. 5, p. 585, 2010

[17] T. Laliberte, L. Birglen, and C. Gosselin, "Underactuation in robotic grasping hands," Machine Intelligence \& Robotic Control, vol. 4, no. 3, pp. 1-11, 2002.

[18] B. Hillberry and A. Hall Jr, "Rolling contact joint," Jan. 13 1976, uS Patent 3,932,045. 